\title{
GETTING DIGITAL DIPLOMACY RIGHT: WHAT QUANTUM THEORY CAN TEACH US ABOUT MEASURING IMPACT?
}

\author{
Corneliu Bjola, Associate Professor of Diplomatic Studies \\ Department of International Development, University of Oxford \\ corneliu.bjola@qeh.ox.ac.uk
}

\begin{abstract}
The issue of maximising the impact and effectiveness of digital diplomacy has moved high on the agenda of many Ministries of Foreign Affairs (MFA). What impact means in the digital context, how to capture it and how best to make use of it are questions that nevertheless remain poorly understood. Drawing heuristically on quantum theory, the article argues that the nature of the impact and the method of measuring it are two facets of the same ontological construct. The very act of measuring shapes the type of impact we may seek to capture. Getting digital diplomacy right paper cannot therefore be reduced to an exercise of fine-tuning quantitative metrics. It involves a more complex approach that takes into account active listening of online conversations, careful prioritisation of short and long-term objectives, hybridisation of online/offline diplomatic agendas, mixed modes of engagement and creative mechanisms of adaptation.
\end{abstract}

Key words: digital diplomacy, digital impact, quantum theory, diplometrics

\section{Introduction}

As cogently indicated by the other contributions in this forum, digital diplomacy is no longer an inchoate field of expertise trying to find its balance in a world challenged and disrupted by the advance of social media technologies. For many Ministries of Foreign Affairs (MFA) around the world, the policy priority has moved on from creating the necessary infrastructure for conducting digital diplomacy to the more ambitious objective of "getting it right". This effort is necessary not only because MFAs find themselves under growing institutional pressure to demonstrate "value for money”, but also because digital competition pushes MFAs to design more effective ways to reach their target audiences. "Diplometrics", the new term coined by MFAs for measuring digital impact, seeks, for instance, to identify suitable combinations of factors (measurable objectives, progress indicators, engagement ratios etc.) that can best track and shape the impact of digital policies and campaigns in real time.

The increased sophistication of digital diplomacy is a welcoming sign of maturity for a profession that did not even exist ten years ago. At the same time, getting digital diplomacy right requires careful conceptual reflection of what impact means in the digital context, how to capture it and how best to make use of it? Drawing heuristically on insights from quantum theory, the article critically examines the ontological and epistemological assumptions underlying the concept of digital diplomatic impact. It thus argues that the nature of the impact and the method of measuring it are very much linked to each other to the extent that the very act of measuring shapes the type of impact we may seek to capture. The paper concludes by introducing five principles of digital conduct that can best maximize the impact and effectiveness of digital diplomacy. 


\section{The Quantum Challenge}

As the fundamental paradigm for describing physical phenomena at the (sub-)atomic level, quantum theory is arguably not the first framework that comes to mind for examining pivotal questions about diplomacy and digital impact. While the authority of quantum theory is quite well established in physics, its reception in social sciences remains much more muted and for good reasons: the properties of quantum systems seem fundamentally inconsistent with the way in which social reality works: material objects dissolve into fields of potentiality, events seem not to have causes, distinct objects seem to remotely influence each other and so on (Wendt 2015, 40).

That being said, a number of studies have recently taken up the challenge of discussing quantum theory outside of physics, both in social science (Haven and Khrennikov 2013) and diplomatic studies (Der Derian 2011). The authors do not explicitly seek to uncover quantum physical effects in social sciences, but rather to heuristically explore the analytical gains that quantum theory may offer for studying various puzzles in psychology, finance, economics and diplomacy. It is in this heuristic light that this article embarks on examining the issue of digital diplomatic impact from a quantum perspective.

By overcoming the linear logical of causality advocated by “classical” approaches, quantum theory promises to offer useful cognitive guides for discussing the ontological and epistemological assumptions underlying the concept of digital diplomatic impact. More specifically, it opens the analytical "door" to the possibility of examining the impact of digital diplomacy not only in discrete terms (A causes B), but also as a continuous process (A and B shape each other). At the same time, it problematizes the influence of the assessment method (e.g., quantitative vs qualitative) on the type of impact to be observed. Two principles of quantum theorising are particularly important to draw on to probe these ideas.

- Wave-particle duality refers to the fact that sub-atomic phenomena can be described both in terms of a particle or a wave, depending on the experimental conditions they find themselves. An electron behaves, for instance, like a wave as long as it is not observed, but it behaves as a particle once it is observed (Wendt 2015, 46). The mathematical theory behind this principle is complicated but it informs what Niels Bohr called "complementarity", the notion that objects have complementary properties which cannot be observed or measured all at the same time (Angelo and Ribeiro 2015). Applied to our case, the duality principle would signal the possibility of conceptualising digital impact not only as a discrete value ("particle”), but also as a more continuous, "wave-like” phenomena. This point will be explored in more detail further below via the distinction between policy outputs vs outcomes.

- Wave function collapse refers to the process by which a wave "collapses" into a particle. From a quantum perspective, when a measurement is performed, the probability of all the possible non-observed outcomes of the wave-like behaviour goes down to zero (Wendt 2015, 47). Applied to our case this means that once we start measuring the impact of a particular digital activity, the potential different outcomes of that activity fade into obscurity. While this observation may sound hardly surprising, it nevertheless calls attention to the fact that the act of measurement is not ontologically neutral: the way in which we measure (e.g., quantitatively or qualitatively) has significant bearing on the type of outcome that we observe. Simply put, our measurements shape how we conceptually refer to digital effects and how we practically employ them. 
The quantum principles described above carry good heuristic value for innovatively unpacking some important ontological and epistemological considerations regarding the concept of digital diplomatic impact. What remains to be seen is whether and how they can enlighten the field of diplomatic impact analysis from an empirical perspective as well. I will address this task by drawing on these principles to make inquiries into the nature of digital impact and the method of measuring it.

\section{What to Measure: Outputs vs Outcomes?}

Does it make sense to conceptualise impact both as a continuous and discrete variable, and if yes, what difference would this make for the way in which diplomats may choose to design their digital activities? Let us consider the example of Iran's Foreign Minister Javad Zarif joined by President Hassan Rouhani in tweeting "Happy Rosh Hashanah" greetings on the occasion of the Jewish New Year's holiday in September 2013. Given the tense diplomatic relationships between Iran and Israel, especially during the tenure of the former president, Mahmoud Ahmadinejad, it is understandable why the diplomatic outreach from Zarif and Rouhani, particularly to the Jewish people, went viral online and was considered a powerful signal for a more engaging and constructive form of diplomacy by Iran (Al-Monitor 2013).

That being said, the issue of whether Zarif and Rouhani's e-greeting has had any impact on Iran's diplomatic relations is not that easy to address as the answer much depends on whether we conceptualise the effects of their action in continuous vs discrete terms. One way to do this is by drawing on the output vs outcome distinction in public policy analysis. The former refers to the products or services provided by the public administration, while the latter subsumes the results that are caused by outputs on social groups (Knoepfel et al. 2011, 11). Paralleling the wave/particle duality principle in quantum theory, outputs and outcomes could be thus considered as complementary phenomena that implicate but do not fully determine each other. The application of classical models of linear causality to unpacking the relationship between outputs and outcomes is therefore fraught with serious difficulties, partly because of the multitude of intervening factors and partly because of the different time scales at which they operate.

Desired outputs in digital diplomacy include, for instance, agenda setting, presence expansion or public engagement, which MFAs use to maximise their digital influence by staying in control of online conversation topics, improving their informational reach with the broader public, and by enabling a two-way or multidirectional communication with their target audiences (Bjola and Jiang 2015). Whether these outputs are instrumental in altering the behaviour of the target audience (outcome) is a different matter. Outputs simply reflect ongoing and short-term consequences of digital activities, while outcomes cover long-term output influences. 

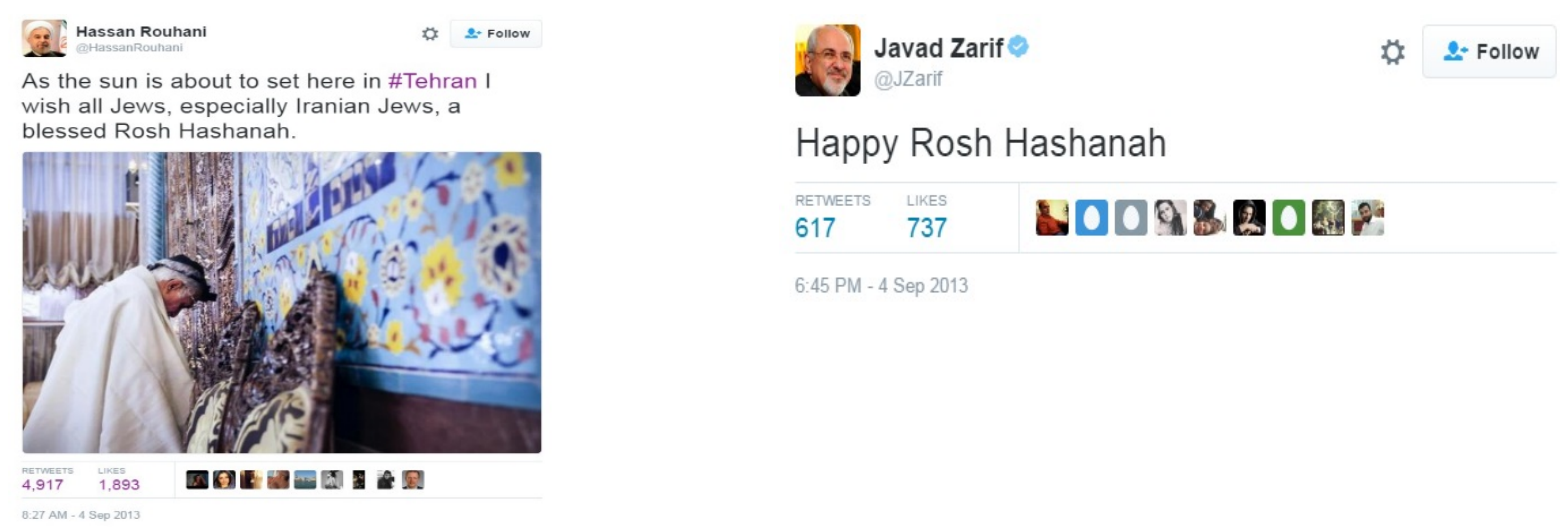

Judged strictly from the "output" perspective, the digital outreach of the Iranian Foreign Minister and President had a reasonably strong impact as it skilfully placed the image of the "New Iran" on the public agenda, generated significant presence and message dissemination on social media channels via retweets and likes, and led to some constructive online exchanges with a number of influential public personalities and journalists. On the broader issue of whether the exchange actually helped shape a new course for Iran's diplomatic relations with other countries ("outcome"), the matter is definitely more controversial. The tweets were not followed by a policy change with Israel, but they might have marginally increased confidence in the two Iranian leaders during their nuclear negotiations with the United States. A dual conceptualisation of impact in terms of outputs vs impact thus helps us gain a more nuanced understanding of the effects of digital diplomacy. At the same time, it exposes a critical tension between the two terms (non-alignment), which might not be easy to resolve. A closer look at the logic behind output vs outcome strategies of diplomatic influence may help shed light on this matter.

Output-first strategy: This approach prioritises the impact of digital outputs at the expense of outcomes on the assumption that causal connections between outputs and outcomes are rather difficult, time consuming and practically irrelevant to establish. As the Finish Director for Public Diplomacy points out, "sometimes it is more important for us to reach a certain subgroup, subculture, genre fans etc. so quality over quantity also does apply in social media [...] if it feels right (to many, not just me) it usually is right” (Theman 2016). In other words, by keep generating good outputs (content, reach, engagement) in a consistent fashion, one should then expect positive outcomes (e.g., perception changes in the target audience) to follow as well at some point. The argument has an appealing logic especially since the fast-paced mode of operation of digital diplomacy leaves little time for conducting laborious assessments of policy impacts that may take months or even years to bear fruit. At the same time, by decoupling outcomes from outputs one runs the risk of spending valuable resources in multiple directions, which may not necessarily be policy convergent, and with probably little to show in terms of improving diplomatic relations with other countries.

Outcome-first strategy: At the opposite pole is the view of subsuming digital outputs to certain predefined outcomes. Consular crisis communication should be particularly amenable to this approach as the goal of assisting nationals in times of terrorist attacks or natural disasters takes precedence above anything else. Making sure that citizens in foreign countries receive timely and accurate information (output) on how to protect themselves from harm during crises (outcome) is a relatively straightforward strategy with clear and tangible effects to track and measure. Policy outcomes thus inform, shape and steer digital outputs. During the Brussels terrorist attack in March 2016 the Spanish MFA found, for instance, that, Twitter was the most effective way of spreading information 
to Spanish nationals. "You will have 1,500 Retweets in approximately 20 minutes and more than 7,000 a little later if the information posted is important” (Femenía 2016). At the same time, one should remain cognisant of the fact that close synchronisation between outcomes and outputs in times of crisis is a challenging task due to a variety of factors including the geographical scope of the crisis, the reliability of ICT networks, the difficulty of fact-checking information in real-time and so on. The best that MFAs and embassies can do in such situations is to make sure they have a predesigned outcome-based crisis strategy in place, which can be promptly activated if the situation arises and the digital outputs of which can be flexibly adjusted during the crisis.

In sum, the causal tension between outputs and outcomes cannot be conclusively solved, but it can be reasonably managed if the context, scale and timeframe of the digital engagement are properly considered. Output-based measurement is particularly important, for instance, for digital activities that involve complex operations, large audiences, and lengthy periods of implementation as it may often happen in digital public diplomacy. Narrower and shorter digital engagements such as situations of crisis communication are more conductive to outcome-based measurements, in which digital outputs are informed by and assessed against tangible policy goals.

\section{How to Measure: Quantitative vs Qualitative Metrics?}

Aside from the thorny issue of the continuous/discrete nature of the digital impact (output vs outcome), the method of measurement is equally important. Quantum theory tells us that measurement has a performative effect on the sub-atomic object under observation by restricting the range of possible conditions the object may find itself. From the perspective of impact analysis, one important conclusion to heuristically follow from this principle is that measurement may shape how we conceptually refer to digital effects and how we practically employ them. In other words, the measurement method (e.g., quantitatively or qualitatively) has significant bearing on the type of digital outcome that we observe.

Let us consider the example of Julie Bishop, the Australian Foreign Minister, who is widely recognised as the leading authority in "emoji-plomacy", the use of pictograms to visually express diplomatic positions and actions (Twiplomacy 2016). Bishop's skilful use of emoji has turned her into a digital diplomatic star, whose tweets and posts are closely followed by a global audience. From a quantitative perspective, her case could not be more successful as the number of retweets, shares, and likes she receives for her Twitter and Facebook entries is often above those of her own Department of Foreign Affairs \& Trade. 


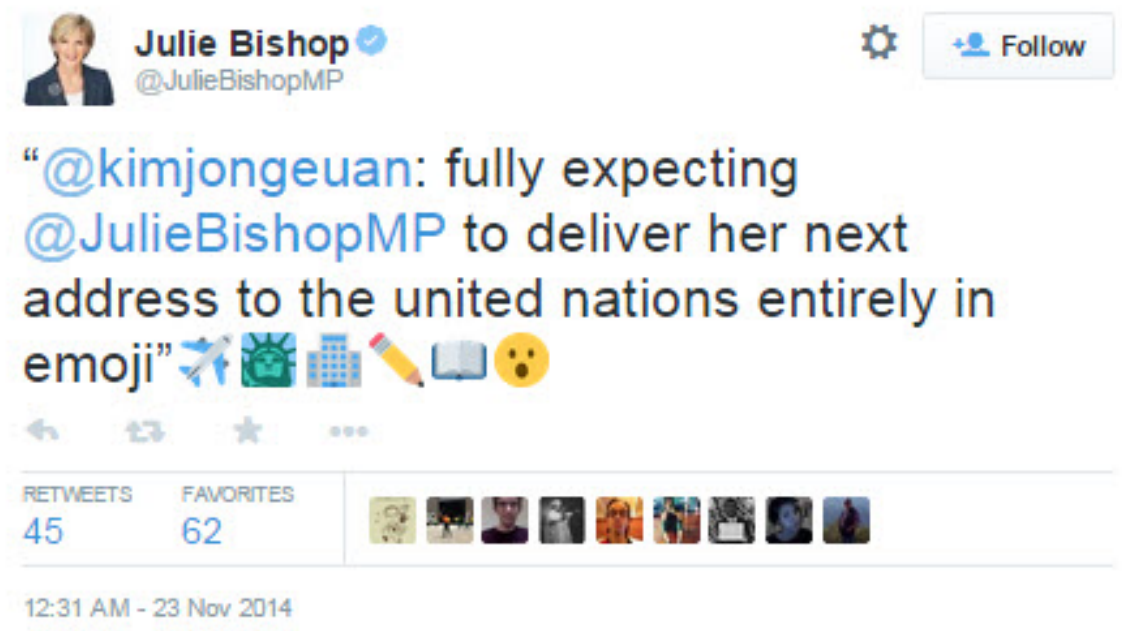

The analytical insights to be gained from using quantitative metrics for assessing the impact of Mrs. Bishop's emoji-based communications, speak to the configurations of factors that allows her to craft messages in a manner that resonate well with a wider audience. Descriptively, large numbers of retweets, shares, and likes signal a positive reception of the message, while low levels of digital interaction indicate apathy if not lack of support for the communication narrative. More sophisticated techniques calculate the lifespans of tweets, determine differences in interaction when posts are in local languages, and compare and contrast the success of various digital diplomacy campaigns (Linzell 2015). Analytically, hashtag, (re)tweet or sentiment analysis offer in-depth investigations of the determinants of digital effectiveness such as trending topics, reachable audiences and resonant themes. Hashtagify, Twitonomy, and Trackur are, for instance, some of the most commonly used analytical tools for quantifying the real-time impact of digital communications, but the number and quality of the software tools available for digital data analysis keep increasing.

There is little doubt that quantitative metrics are critically necessary for making sure that one's digital communication strategy is properly working by reaching the relevant audience in a timely and effective fashion. However, quantitative metrics do not tell the full story. Actually, the focus on quantitative metrics renders the question of impact into a matter of measuring clearly defined expectations of how digital engagement should work and be effective. In other words, one generally knows what to expect from digital diplomatic activities (e.g., well and broadly received messages) and then uses quantitative metrics to fine-tune the pathway to the expected impact. The contentious issue is not that quantitative metrics are inaccurate, but rather that, as suggested above by the wave collapse principle, the quantitative method of measuring impact comes with a heavy in-built bias towards a specific type of pre-defined outcome (message dissemination).

However, what if the digital impact we would like to assesses is not a priori defined? Put differently, what if we seek to localize effects of digital diplomacy, aside from the issue of message dissemination that quantitative metrics capture so well, but we do not know where and how to look for them? This is where qualitative methods could prove particularly useful due to their proclivity for contextual- and inductive-based research. From a qualitative perspective, the impact of digital diplomacy is "discovered" not confirmed. The assessment effort is not directed at understanding how well the digital message travel, as in the case of quantitative metrics, but at uncovering potential relationship patterns that digital activities may make possible. Going back to our example, the qualitative impact of Mrs. Bishop’s emoji-diplomacy would rest, for instance, not on the 
effectiveness of her message with the online public, but on whether the type of digital engagement she practices is conducive to creating online relationships of diplomatic relevance that did not exist prior to her digital interventions.

Immersing oneself in the context of digital interactions is the first step towards qualitatively unpacking the impact of digital diplomatic activities. This requires a sustained effort to directly observe the interaction patterns to result from digital diplomatic activities. These may include homophilic relationships by which individuals bond with status- or value-minded persons (Lazarsfeld and Merton 1954) leading to the formation of digital “echo-chambers” (Bjola 2016), sub-networks of mutual influence by which members promote or reinforce each other's messages and positions , "communities of practice” interested in exchanging views about methods of engaging in digital diplomacy and so on. Social network analysis (Carrington and Scott 2011) is a particularly good approach for mapping and examining such relationships as it offers a cluster of useful indicators such as degree centrality, path distance, and closeness (Kumar 2013) for identifying influencing nodes in a network, assessing the nature of the relationships between them and for tracing their patterns of engagement.

To sum up, the very act of measuring shapes the type of impact we seek to capture. Quantitative metrics predispose us to define impact mainly as message dissemination, while qualitative indicators direct our attention to tracing relationship patterns that digital activities may make possible. A number of interesting questions may follow from this conclusion: under what circumstances would make sense to prioritise quantitative and qualitative measures over each other? What are the benefits and limitations of using mixed indicators for measuring impact? What forms of digital empowerment might follow from the application of quantitative vs qualitative methods and what kind of ethical considerations should accordingly be taken into account?

\section{Getting it Right: Five Principles of Impactful Digital Diplomacy}

The heuristic application of two quantum principles to examining the nature and method of digital diplomatic impact suggests that no "silver bullet" solution is likely available. The quest for an integrated pathway to impact that combines outputs and outcomes with qualitative and qualitative indicators into a universal, one-size-fits-all formula is not only conceptually flawed but also practically unfeasible. The more sensible alternative is to understand how the trade-offs between these various factors can be constructively negotiated. The following five principles of digital conduct could best accomplish this task.

- Listening - actively monitoring online conversations is a critical step towards developing an impactful digital diplomacy. Quantitative metrics may be used for tracing rising trends and resonant themes of discussion, while qualitative research can help uncover networks of influence of relevance for one's diplomatic objectives. Passively following popular trends and influencers is not, of course, the main rationale for social media listening, but rather the necessity to make sure that the pathway to impact is not obstructed by avoidable points of resistance.

- $\quad$ Prioritisation - no digital strategy can succeed without clearly defined short-term and long term objectives. However, as pointed out above, the context of the digital activity largely defines the nature of the objectives one should seek to pursue. Complex operations involving 
large and multiple audiences over long periods of time are more amenable, for instance, to incremental, output-based measurements as opposed to broad and ambitious long-term outcomes that are difficult if not impossible to deliver.

- Hybridisation: while distinct in method, digital diplomacy does not operate independently of traditional diplomatic channels. Borrowing from Goffman (1959), the online "front-stage" and offline diplomatic "back-stage" need to be well coordinated in order for each to be effective and successful. This means that digital outputs and outcomes and cannot be allowed to substantially diverge or contradict objectives set for traditional diplomacy.

- Engagement: a key advantage of digital diplomacy is the possibility of directly reaching large audiences in real-time. Engagement, however, may vary from simple modes of info broadcast to more sophisticated forms of diplomatic advocacy. Quantitative metrics can help keep track of the short-term impact of digital engagements, but qualitative methods can reveal patterns leading to the development of long-term digital connections, relationships and networks.

- Adaptation: digital diplomats do not operate in a static environment as their actions are constantly influenced, shaped and constrained by the actions of other digital players. As a result, quantitative and qualitative measurement methods must be designed with a certain level of inbuilt flexibility to allow them to capture changing patterns of interactions with and inside the target audience.

The five principles described above offer a comprehensive framework for developing strategies that can maximise the impact and effectiveness of digital diplomacy, but not in a linear, deterministic fashion. None of these principle is likely to be sufficient by itself to decisively improve digital impact, but a combination of them should be able to accomplish this if they operate together and reinforce each other. Actively listening should lead to informed prioritisation and coherent hybridisation, which in turn may boost engagement and improve adaptation. By contrast, failure to take note of these principles would likely have a negative impact on the effectiveness of digital activities. Faulty listening and ambitious prioritisation would likely lead to deficient engagement, while conflicting hybridisation could undermine adaptation efforts. Fig 1 visually describes how the five principles can be used as an impact assessment matrix for digital diplomacy. High impact (5 on the scale) would correspond to a perfect alignment of the five principles, while low impact (1 on the scale) would be the result of their complete neglect. Most of the cases, however, like the ones mentioned above, would likely fit in between the two opposite ends of the spectrum ( 2 or 3 on the scale), featuring good levels of engagement and adaptation but low to medium interest in listening, prioritisation or hybridisation. 
Fig 1: Impact Assessment Matrix for Digital Diplomacy

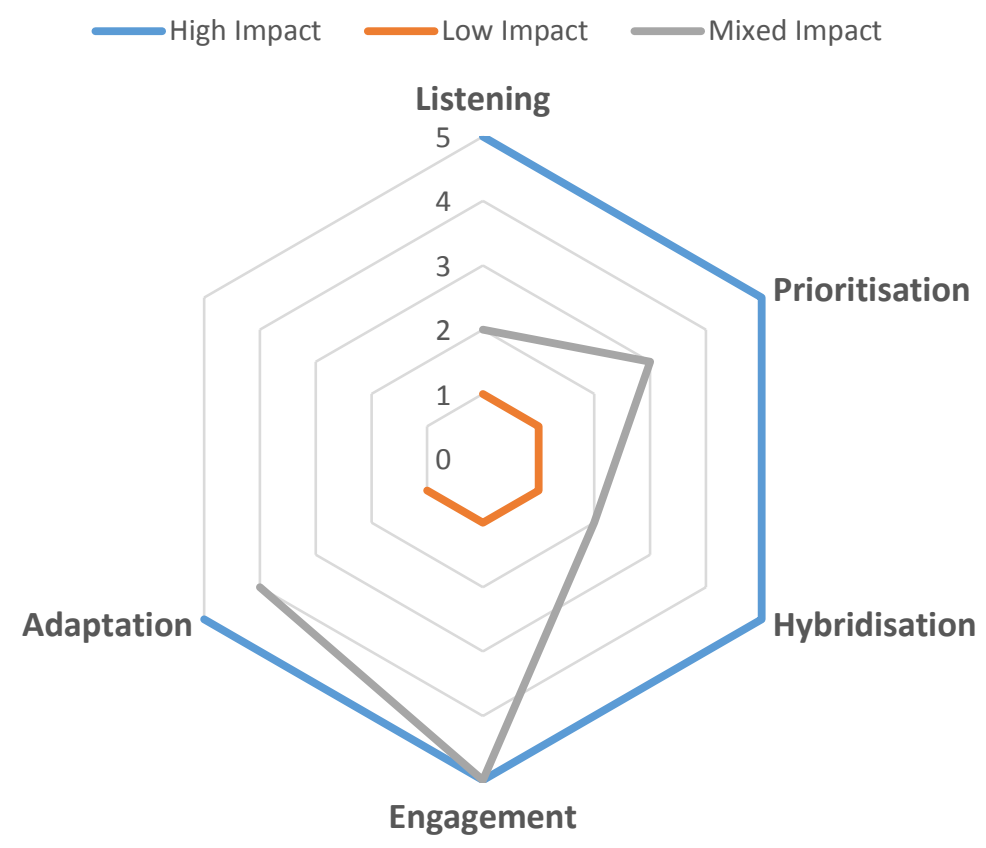

To conclude, the question of digital diplomatic impact comes with some heavy ontological and epistemological assumptions that need to be first unpacked before the question can be properly addressed. Drawing heuristically on quantum theory, the paper has argued that the nature of the impact and the method of measuring it are two facets of the same ontological construct. The very act of measuring shapes the type of impact we may seek to capture. Getting digital diplomacy right paper cannot therefore be reduced to an exercise of fine-tuning quantitative metrics. It involves a more complex approach that takes into account active listening of online conversations, careful prioritisation of short and long-term objectives, hybridisation of online/offline diplomatic agendas, mixed modes of engagement and creative mechanisms of adaptation. 


\section{References:}

Al-Monitor. 2013. "Iran's Rosh Hashana Twitter diplomacy stirs amazement, disbelief." Last Modified Sept 6 Accessed June 2. http://www.al-monitor.com/pulse/iw/contents/articles/originals/2013/09/iranrouhani-zarif-khamenei-israel-nuclear-twitter-psaki-us.html.

Angelo, R. M., and A. D. Ribeiro. 2015. "Wave-Particle Duality: An Information-Based Approach." Foundations of Physics 45 (11):1407-1420. doi: 10.1007/s10701-015-9913-6.

Bjola, Corneliu. 2016. "Digital Diplomacy and the Bubble Effect: The NATO Scenario." USC Center on Public Diplomacy, Last Modified Mar 8 Accessed June 17. http://uscpublicdiplomacy.org/blog/digitaldiplomacy-and-bubble-effect-nato-scenario.

Bjola, Corneliu, and Lu Jiang. 2015. "Social Media and Public Diplomacy: A Comparative Analysis of the Digital Diplomatic Strategies of the EU, U.S. and Japan in China." In Digital diplomacy : theory and practice, edited by Corneliu Bjola and Marcus Holmes, pages cm. Milton Park, Abingdon, Oxon ; New York: Routledge.

Carrington, Peter J., and John Scott. 2011. The SAGE handbook of social network analysis. London: SAGE.

Der Derian, James. 2011. "Quantum Diplomacy, German-US Relations and the Psychogeography of Berlin." The Hague Journal of Diplomacy 6 (3-4):373-392. doi: doi:http://dx.doi.org/10.1163/187119111X598152.

Femenía, Consuelo. 2016. "Digital diplomacy as a team work tester." Twiplomacy, Last Modified May 31 Accessed June 2. http://twiplomacy.com/blog/digital-diplomacy-as-a-team-work-tester/.

Goffman, E. 1959. The Presentation of Self in Everyday Life: Doubleday.

Haven, Emmanuel, and A. TU Khrennikov. 2013. Quantum social science. Cambridge ; New York: Cambridge University Press.

Knoepfel, Peter, Corinne Larrue, Michael Hill, and Frédéric Varone. 2011. Public policy analysis. Bristol: Bristol : Policy Press.

Kumar, Shamanth. 2013. Twitter data analytics. New York: Springer.

Lazarsfeld, P. F., and R. K. Merton. 1954. "Friendship as a social process: A substantive and methodological analysis." In Freedom and Control in Modern Society, edited by M. Berger, T. Abel and C. Page, 18-66. Van Nostrand.

Linzell, Steven. 2015. "Digital evaluation: crunching the numbers with Ripjar." Foreign and Commonwealth Office, Last Modified June 23 Accessed June 12.

http://blogs.fco.gov.uk/stevenlinzell/2015/06/23/digital-evaluation-crunching-the-numbers-withripjar/.

Theman, Petra. 2016. "Quality over Quantity also applies in Social Media." Twiplomacy, Last Modified May 31 Accessed June 2. http://twiplomacy.com/blog/quality-over-quantity-also-applies-in-socialmedia/.

Twiplomacy. 2016. "Emoji Diplomacy - a new diplomatic sign language." Last Modified April 8 Accessed June 3. http://twiplomacy.com/blog/emoji-diplomacy-a-new-diplomatic-sign-language/.

Wendt, Alexander. 2015. Quantum mind and social science : unifying physical and social ontology. Cambridge, United Kingdom ; New York: Cambridge University Press. 